\title{
ON THE SOLUTION OF A TRANSCENDENTAL EQUATION ARISING IN THE THEORY OF SCATTERING BY A DIELECTRIC CYLINDER*
}

\author{
BY \\ W. STREIFER AND R. D. KODIS \\ Brown University
}

1. Introduction. It is well-known that a number of effective procedures for dealing with the problem of scattering by a large dielectric (penetrable) cylinder begin by transforming the solution obtained by the usual separation of variables (Fourier series of angular eigenfunctions) into an infinite series of integrals of the radial eigenfunctions. The transformation may be carried out in a variety of ways, two of which have been widely used: the Poisson sum formula as applied by Wu [1], and the so-called Watson transformation [2]. These procedures are similar to those discussed by Titchmarsh [3] and Marcuvitz [4]. The investigations of Franz and his co-workers, for example, have been based on this transformation [5]-[9].

An important feature of the representation is that some of the integrals have stationary phase points. It has been shown by Beckmann and Franz [6] and by Rubinow [10] that these contributions to the field correspond to the rays of geometric optics. Other integrals, however, have no points of stationary phase; they represent diffraction effects which can be evaluated by finding the poles of the integrand and computing the residues. The equations which must be solved to carry out this program for the problem of scattering by a dielectric cylinder have the form

$$
k_{2} \frac{H_{\nu}^{(1)^{\prime}}\left(k_{2} a\right)}{H_{v}^{(1)}\left(k_{2} a\right)}-k_{1} \frac{H_{\nu}^{(2)^{\prime}}\left(k_{1} a\right)}{H_{v}^{(2)}\left(k_{1} a\right)}=0
$$

and

$$
k_{2} \frac{H_{\nu}^{(1)^{\prime}}\left(k_{2} a\right)}{H_{\nu}^{(1)}\left(k_{2} a\right)}-k_{1} \frac{J_{\nu}^{\prime}\left(k_{1} a\right)}{J_{\nu}\left(k_{1} a\right)}=0,
$$

where " $a$ " is the cylinder radius, and $k_{2}$ is the wave number of the infinite medium containing the dielectric cylinder of wave number $k_{1}$. The first equation has been considered by Franz and Beckmann [7]; however, their results are mainly qualitative and not completely correct.

The asymptotic solution of the first equation is outlined in this paper for large, real values of $k_{1} a$ and $k_{2} a$, with $k_{1} a<k_{2} a$. This choice was made in the hope that the results would be useful in problems of scattering from ionized gas columns, such as plasmas or meteor trials. The solution with $k_{1} a>k_{2} a$ or with $k_{1} a$ complex may also be obtained by the methods developed here as may the solutions of similar equations arising in problems of scattering by spheres.

The second equation will be considered elsewhere.

2. Solution. The problem is to determine, for given $x$ and $y$, the values of $\nu$ which satisfy the equation

$$
x \frac{H_{\nu}^{(1)^{\prime}}(x)}{H_{\nu}^{(1)}(x)}-y \frac{H_{\nu}^{(2)^{\prime}}(y)}{H_{\nu}^{(2)}(y)}=0 ; \quad x, y \quad \text { real } \gg 1,
$$

*Received March 1, 1963. 


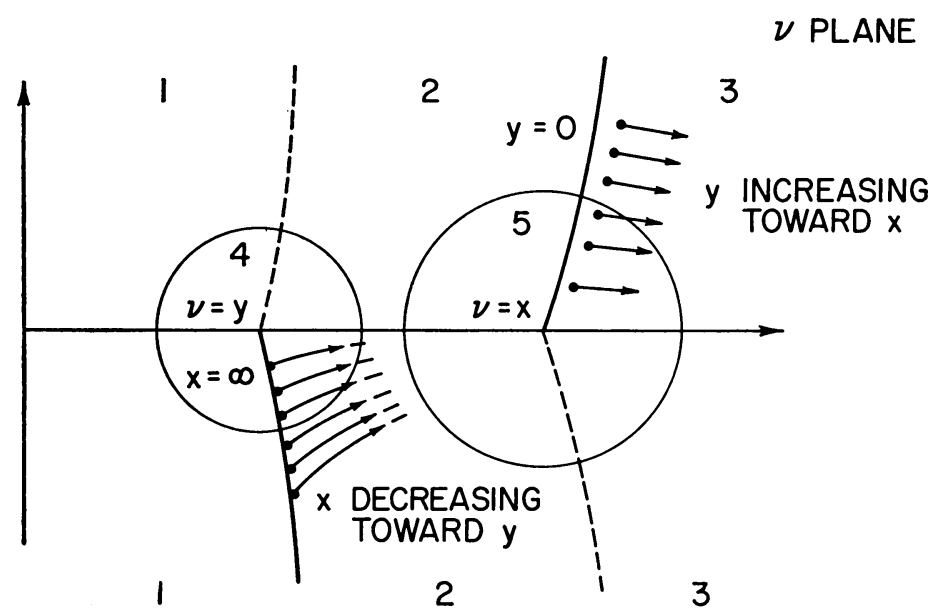

Fig. 1. Regions and root locations in the right half plane.

where $x=k_{2} a, y=k_{1} a$ and $y<x$. Since the replacement of $\nu$ by $-\nu$ leaves equation (1) unchanged, its roots must be symmetrically located with respect to the origin. It is therefore necessary to examine the equation only in half the complex $\nu$ plane, and we arbitrarily select the right half plane, $\operatorname{Re}(\nu)>0$.

The solutions will be developed as asymptotic series in $x$ or $y$ by means of appropriate approximations for the Hankel functions. Since each of these forms can be used only within certain ranges of the parameters $\nu, x$, and $y$, the right half $\nu$ plane must be divided into five parts as shown in Figure 1, and suitable expansions developed for each region. Regions 1,2, and 3 are separated by the lines which contain the zeros of the Hankel functions and their derivatives (see Appendix A). Regions 4 and 5 are circular regions with radii $|\nu-y|=0\left(y^{1 / 3}\right)$ and $|\nu-x|=0\left(x^{1 / 3}\right)$. Since the order symbol as it is usually defined [11] does not specify a particular constant, regions 4 and 5 do not have specific boundaries. We can distinguish two cases:

(1) $x-y=0(x)$. Regions 4 and 5 do not overlap;

(2) $x-y=0\left(x^{1 / 3}\right)$. The two regions do overlap.

Case 1: $x-y=0(x)$. In this case the circular regions are well separated, and the roots of equation (1) for large $x$ and $y$ are located in the vicinity of the solid curves of Figure 1. To see this, we note that far from these curves, when $|\nu-x|=0(x)$ and $|\nu-y|=0(y)$, both Hankel functions may be accurately represented by a single exponential function (see Appendix A). If the appropriate asymptotic forms, (A.6) and (A.7), are substituted in equation (1), the result in all cases is

$$
\left(\nu^{2}-x^{2}\right)^{1 / 2}-\left(\nu^{2}-y^{2}\right)^{1 / 2}+0(1)=0 .
$$

Transposing, squaring, and employing $x-y=0(x)$ yields $x^{2}-y^{2}=0(x)$ or $0(x)^{2}=0(x)$, which cannot be an equality for large $x$. It follows that for large $x$ and $y$ no roots exist which satisfy the order conditions stated above.

In region 4, where $|\nu-y|=0\left(y^{1 / 3}\right),|\nu-x|=0(x)$ and $x-y=0(x)$, the expansion for the roots of equation (1) can be found by means of the asymptotic forms:

$x \frac{H_{\nu}^{(1)}(x)}{H_{\nu}^{(1)}(x)} \sim i\left(x^{2}-\nu^{2}\right)^{1 / 2}-\frac{x^{2}}{2\left(x^{2}-\nu^{2}\right)}+\frac{i x^{2}}{8\left(x^{2}-\nu^{2}\right)^{3 / 2}}\left(1-\frac{5 \nu^{2}}{\nu^{2}-x^{2}}\right)+0\left(x^{-2}\right)$ 
and

$y \frac{H_{\nu}^{(2)}(y)}{H_{\nu}^{(2)}(y)} \sim-\frac{1}{3}+\frac{y}{p(y, \nu)} \frac{\partial p(y, \nu)}{\partial y}-y e^{\pi i / 3} \frac{\partial q(y, \nu)}{\partial y} \frac{A i^{\prime}\left(-q e^{\pi i / 3}\right)}{A i\left(-q e^{\pi i / 3}\right)}+0\left(x^{-2 m / 3}\right)$.

In the latter representation $p(y, \nu)$ and $q(y, \nu)$ are the Schöbe polynomials of (A.9a) and (A.9b) truncated at $m=3$ so that the neglected terms in both approximations are of the same order. $A i(\eta)$ is the Airy function of the first kind (Appendix B) and the prime indicates differentiation with respect to the argument. To this degree of approximation, equation (1) takes the form

$$
\frac{A_{i}^{\prime}\left(-q e^{\pi i / 3}\right)}{A i\left(-q e^{\pi i / 3}\right)}=\Gamma
$$

with

$$
\begin{aligned}
\Gamma=\frac{i e^{-\pi i / 3}}{\partial_{y} q}\left[\left(\frac{1}{N^{2}}-\frac{\nu^{2}}{y^{2}}\right)^{1 / 2}+\right. & \frac{i x}{2 N\left(x^{2}-\nu^{2}\right)}-\frac{i}{3 y}+\frac{i \partial_{y} p}{p} \\
& \left.\quad+\frac{x}{8 N\left(x^{2}-\nu^{2}\right)^{3 / 2}}\left(1-\frac{5 \nu^{2}}{\nu^{2}-x^{2}}\right)\right]+0\left(x^{-8 / 3}\right)
\end{aligned}
$$

and $N=y / x$.

To solve (2a) subject to (2b) and the definition of $q$, we use the fact that all derivatives of $A i(\eta)$ may be expressed as appropriate polynomials in $\eta$ multiplied by $A i(\eta)$ and $A i^{\prime}(\eta)$ (see Appendix B). We then seek solutions of (2a) for large $\Gamma$, i.e. $N=y / x \rightarrow 0$, which implies that the relative dielectric constant of the cylinder is small compared to unity. Since $A i^{\prime}(\eta)$ is finite for all finite $\eta$ [12], the left side of (2a) is unbounded only at the zeros of $A i(\eta)$. Finite zeros of $A i(\eta)$ occur only when $\eta$ is real and negative. We therefore make the change of variable

$$
\eta=-q e^{\pi i / 3}
$$

and expand $A i^{\prime}(\eta)$ and $A i(\eta)$ in Taylor series about $\eta_{0}$ employing equation (B.3) with $A i\left(\eta_{0}\right)=0$ and $\delta \eta=\eta-\eta_{0}$. Equation (2a) becomes

$$
\frac{\left\{1+\frac{\eta_{0}}{2}(\delta \eta)^{2}+\frac{1}{3}(\delta \eta)^{3}+\frac{\eta_{0}^{2}}{24}(\delta \eta)^{4}+\frac{\eta_{0}}{20}(\delta \eta)^{5}+\frac{\left(10+\eta_{0}^{3}\right)}{6 !}(\delta \eta)^{6}+\frac{12 \eta_{0}^{2}}{7 !}(\delta \eta)^{7}+\cdots\right\}}{\delta \eta\left\{1+\frac{\eta_{0}}{6}(\delta \eta)^{2}+\frac{1}{12}(\delta \eta)^{3}+\frac{\eta_{0}^{2}}{5 !}(\delta \eta)^{4}+\frac{\eta_{0}}{5 !}(\delta \eta)^{5}+\frac{\left(10+\eta_{0}^{3}\right)}{7 !}(\delta \eta)^{6}+\frac{12 \eta_{0}^{2}}{8 !}(\delta \eta)^{7}+\cdots\right\}}=\Gamma
$$

This equation can be solved for $\delta \eta$ by assuming a power series solution in $1 / \Gamma$ and evaluating its coefficients, or by making a Taylor expansion about the origin of the $1 / \Gamma$ plane. The resulting series is

$$
\delta \eta=\frac{1}{\Gamma}+\frac{\eta_{0}}{3}\left(\frac{1}{\Gamma}\right)^{3}+\frac{1}{4}\left(\frac{1}{\Gamma}\right)^{4}+\frac{\eta_{0}^{2}}{5}\left(\frac{1}{\Gamma}\right)^{5}+\frac{7 \eta_{0}}{18}\left(\frac{1}{\Gamma}\right)^{6}+\left(\frac{\eta_{0}^{3}}{7}+\frac{5}{28}\right)\left(\frac{1}{\Gamma}\right)^{7}+\cdots .
$$

Using (3) and the definition of $\delta \eta$, we find that

$$
\begin{aligned}
q(y, \nu)=-\eta_{0} e^{-\pi i / 3} & +i\left(\frac{1}{\gamma}\right)+\frac{i \eta_{0}}{3} e^{-\pi i / 3}\left(\frac{1}{\gamma}\right)^{3}+\frac{1}{4}\left(\frac{1}{\gamma}\right)^{4} \\
& +\frac{i \eta_{0}^{2}}{5} e^{-2 \pi i / 3}\left(\frac{1}{\gamma}\right)^{5}+\frac{7 \eta_{0}}{18} e^{-\pi i / 3}\left(\frac{1}{\gamma}\right)^{6}-i\left(\frac{\eta_{0}^{3}}{7}+\frac{5}{28}\right)\left(\frac{1}{\gamma}\right)^{7}+\cdots
\end{aligned}
$$


where

$$
\gamma=-i e^{-\pi i / 3} \Gamma
$$

Equation (6) may be solved for $\nu$ by an iterative procedure. The zeroth-order approximation is found by dropping all terms on the right except the first, with the result

$$
q_{0}(y, \nu)=-\eta_{0} e^{-\pi i / 3}+0\left(y^{-1 / 3}\right) .
$$

On the left we use the first term of expansion (A.9b),

$$
q_{0}(y, \nu)=\xi+0\left(y^{-2 / 3}\right)
$$

where

$$
\xi=(\nu-y)(2 / y)^{1 / 3}
$$

It follows that

$$
\nu^{(0)}=y-\eta_{0} e^{-\pi i / 3}(y / 2)^{1 / 3}+0(1) .
$$

This approximation coincides with the zeroth-order asymptotic roots of $H_{\nu}^{(2)}(y)=0$ in the lower half plane of figure 1. Higher order approximations for $\nu$ may be obtained by substituting $\nu^{(0)}$ in equation (2b) for $\Gamma$. This enables one to calculate a first order approximation for the right hand side of (6). Similarly, a first order approximation for the left hand member, using (A.9b), yields an equation which can be solved for $\nu^{(1)}$. Thus

$$
\begin{aligned}
\frac{1}{\gamma}=-\partial_{y} q\left(1 / N^{2}-1\right)^{-1 / 2}\left\{1-\frac{\eta_{0}}{2} e^{-\pi i / 3}\left(\frac{1}{\gamma_{0}}\right)^{2}+0\left(y^{-1}\right)\right\} \\
\quad=\frac{1}{\gamma_{0}}\left\{1-\frac{\eta_{0}}{2} e^{-\pi i / 3}\left(\frac{1}{\gamma_{0}}\right)^{2}-\frac{2 \eta_{0}}{15} e^{-x i / 3}\left(\frac{2}{y}\right)^{2 / 3}+0\left(y^{-1}\right)\right\}
\end{aligned}
$$

where

$$
\gamma_{0}=\left(1 / N^{2}-1\right)^{1 / 2}(y / 2)^{1 / 3}=0\left(y^{1 / 3}\right)
$$

and so

$$
\begin{aligned}
q_{1}(y, \nu)=-\eta_{0} e^{-\pi i / 3}+i\left(\frac{1}{\gamma_{0}}\right)-i \frac{2 \eta_{0}}{15} e^{-\pi i / 3}\left(\frac{1}{\gamma_{0}}\right)\left(\frac{2}{y}\right)^{2 / 3} & \\
& -i \frac{\eta_{0}}{6} e^{-\pi i / 3}\left(\frac{1}{\gamma_{0}}\right)^{3}+0\left(y^{-4 / 3}\right) .
\end{aligned}
$$

Substituting the assumed expression,

$$
\nu^{(1)}=\nu^{(0)}+d_{0}+d_{1}\left(\frac{2}{y}\right)^{1 / 3}+d_{2}\left(\frac{2}{y}\right)^{2 / 3}+0\left(y^{-1}\right),
$$

in $(9 \mathrm{~b})$ and the latter in

$$
q_{1}(y, \nu)=\xi-\frac{1}{60} \xi^{2}\left(\frac{2}{y}\right)^{2 / 3}+0\left(y^{-4 / 3}\right),
$$

we obtain 


$$
\begin{aligned}
q_{1}(y, \nu)=-\eta_{0} e^{-\pi i / 3}+d_{0}\left(\frac{2}{y}\right)^{1 / 3} & +d_{1}\left(\frac{2}{y}\right)^{2 / 3}+d_{2}\left(\frac{2}{y}\right)+ \\
& -\frac{\eta_{0}^{2}}{60} e^{-2 \pi i / 3}\left(\frac{2}{y}\right)^{2 / 3}+\frac{d_{0} \eta_{0}}{30} e^{-x_{i} / 3}\left(\frac{2}{y}\right)+0\left(y^{-4 / 3}\right) .
\end{aligned}
$$

Equating the coefficients of equal powers in (11) and (13), we find that

$$
\begin{aligned}
\nu^{(1)}=y-\eta_{0} e^{-x i / 3}\left(\frac{y}{2}\right)^{1 / 3} & +i\left(\frac{1}{\gamma_{0}}\right)\left(\frac{y}{2}\right)^{1 / 3}+\frac{\eta_{0}^{2}}{60} e^{-2 \pi i / 3}\left(\frac{2}{y}\right)^{1 / 3}+ \\
& -\frac{i \eta_{0}}{6} e^{-\pi i / 3}\left(\frac{1}{\gamma_{0}}\right)^{3}\left(\frac{y}{2}\right)^{1 / 3}-\frac{i \eta_{0}}{6} e^{-x i / 3}\left(\frac{1}{\gamma_{0}}\right)\left(\frac{2}{y}\right)^{1 / 3}+0\left(y^{-1}\right) .
\end{aligned}
$$

This process may be carried as far as time and the initial accuracy of equations (2a) and (2b) will allow. Two additional iterations using (A.6) give an expansion to $0\left(y^{-2}\right)$ :

$$
\begin{aligned}
\nu=y & -\eta_{0} e^{-x_{i} / 3}\left(\frac{y}{2}\right)^{1 / 3}+\frac{\eta_{0}^{2}}{60} e^{-2 \pi i / 3}\left(\frac{2}{y}\right)^{1 / 3}-\left[\frac{\eta_{0}^{3}}{1400}+\frac{1}{140}\right]\left(\frac{2}{y}\right) \\
& -e^{-\pi i / 3}\left[\frac{281 \eta_{0}^{4}}{4536000}+\frac{29 \eta_{0}}{12600}\right]\left(\frac{2}{y}\right)^{5 / 3} \\
& +i\left(\frac{1}{N^{2}}-1\right)^{-1 / 2} \\
& -\frac{i \eta_{0}}{6} e^{-\pi_{i} / 3}\left\{\left(\frac{1}{N^{2}}-1\right)^{-3 / 2}+\left(\frac{1}{N^{2}}-1\right)^{-1 / 2}\right\}\left(\frac{2}{y}\right)^{2 / 3} \\
& +\frac{i \eta_{0}^{2}}{20} e^{-2 \pi i / 3}\left\{\frac{3}{2}\left(\frac{1}{N^{2}}-1\right)^{-5 / 2}+\frac{13}{9}\left(\frac{1}{N^{2}}-1\right)^{-3 / 2}-\frac{1}{18}\left(\frac{1}{N^{2}}-1\right)^{-1 / 2}\right\}\left(\frac{2}{y}\right)^{4 / 3} \\
& -\frac{\eta_{0}}{36} e^{-\pi i / 3}\left\{\left(\frac{1}{N^{2}}-1\right)^{-3}+2\left(\frac{1}{N^{2}}-1\right)^{-2}+\left(\frac{1}{N^{2}}-1\right)^{-1}\right\}\left(\frac{2}{y}\right)^{5 / 3} \\
& +\frac{i}{8}\left\{\frac { \eta _ { 0 } ^ { 3 } } { 2 } \left[\frac{5}{7}\left(\frac{1}{N^{2}}-1\right)^{-7 / 2}+\frac{21}{\Delta 5}\left(\frac{1}{N^{2}}-1\right)^{-5 / 2}+\frac{23}{175}\left(\frac{1}{N^{2}}-1\right)^{-3 / 2}\right.\right. \\
& \left.+\frac{1}{175}\left(\frac{1}{N^{2}}-1\right)^{-1 / 2}\right]-\frac{5}{28}\left(\frac{1}{N^{2}}-1\right)^{-7 / 2}+\frac{3}{10}\left(\frac{1}{N^{2}}-1\right)^{-5 / 2} \\
& \left.\left.+\frac{13}{140}\left(\frac{1}{N^{2}}-1\right)^{-3 / 2}-\frac{1}{35}\left(\frac{1}{N^{2}}-1\right)^{-1 / 2}\right]\right\}\left(\frac{2}{y}\right)^{2}+0\left(y^{-7 / 3}\right) .
\end{aligned}
$$

Formula (15) separates naturally into two parts. The first of these does not depend on the refractive index, $N$, of the cylinder; indeed it is all that remains as $N \rightarrow 0(x \rightarrow \infty)$. Examining equation (1) in this limit, one concludes that the first part of the series must be the asymptotic solution of $H_{v}^{(2)}(y)=0$. Comparison of the initial terms of (15) with the results of Sommerfeld [13] and Franz and Galle [5] verifies this conclusion. It follows that for each $\eta_{0}$, with large enough $y$, this part of the series converges to roots which are located on the curve separating regions 1 and 2 in the lower half plane of Figure 1 . The second part of the asymptotic series for $\nu$ contains the effect of finite $N$. For any predetermined $\eta_{0}$ and $N$ the series is only semi-convergent and must be truncated at its smallest term. 


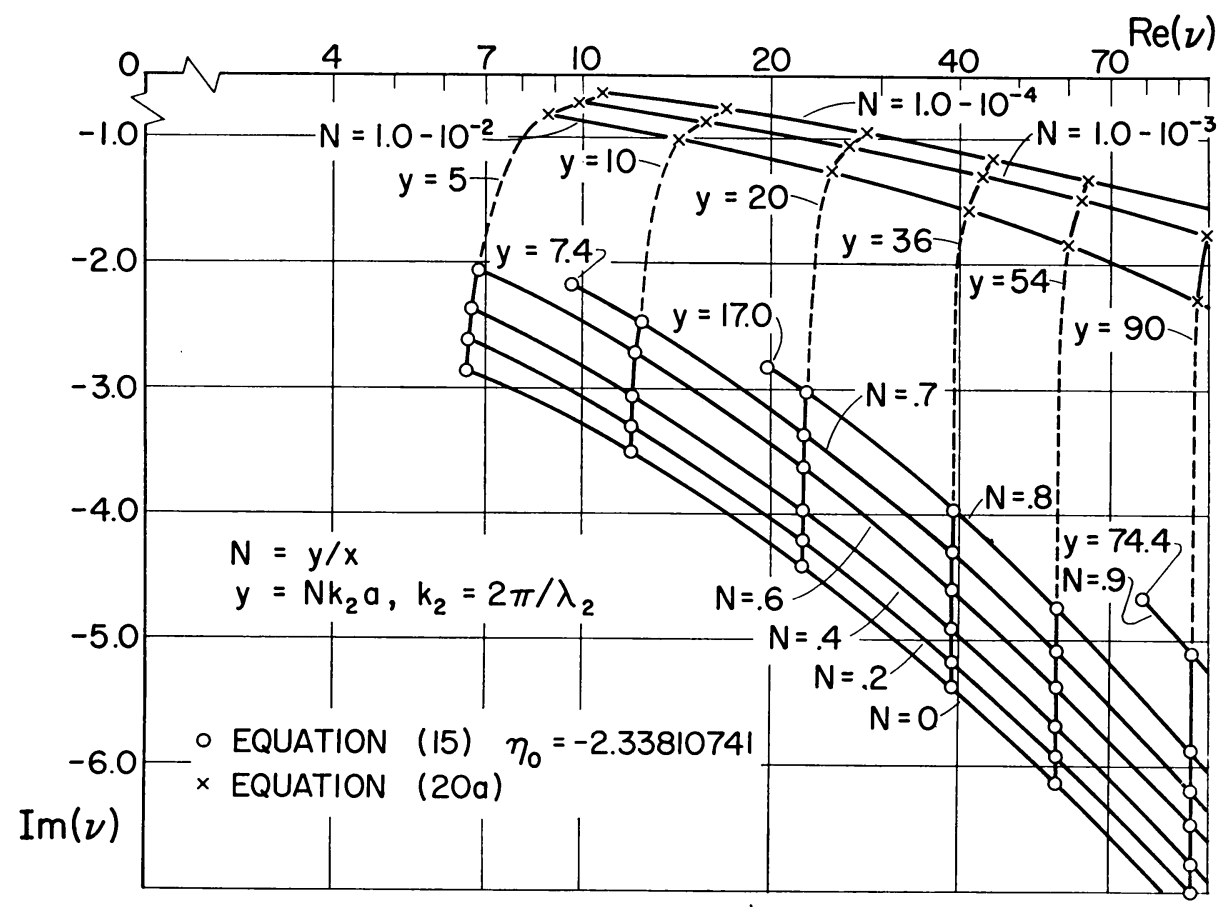

FIG. 2. The first root of $x \frac{H_{\nu}^{(1) '}(x)}{H_{\nu}^{(1)}(x)}-y \frac{H_{\nu}^{(2) '}(y)}{H_{\nu}^{(2)}(y)}=0$ near $|\nu|=y$.

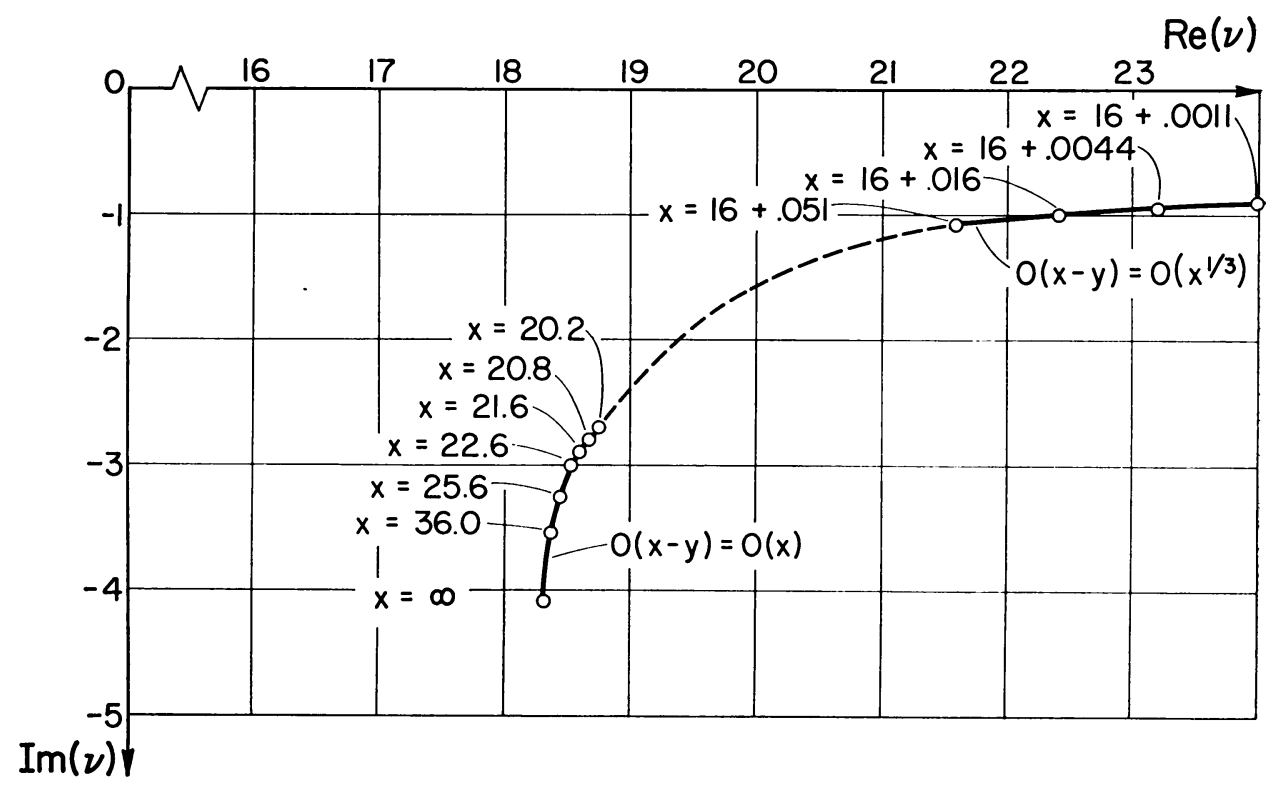

FIG. 3. Locus of the first root near $|\nu|=y$,

$x \frac{H_{\nu}^{(1)}(x)}{H_{\nu}^{(1)}(x)}-y \frac{H_{\nu}^{(2)}(y)}{H_{\nu}^{(2)}(y)}=0$ for $y=16$. 
In the derivation of (15) the binomial, $\left[1+\eta_{0} e^{-\pi i / 3} / \gamma_{0}^{2}\right]^{1 / 2}$, was expanded in a power series on the assumption that $\gamma_{0}^{2}>\left|\eta_{0}\right|$ where $\gamma_{0}=\left(1 / N^{2}-1\right)^{1 / 2}(y / 2)^{1 / 3}$. For a given index of refraction and for each root, this condition gives the limiting value of $y$ (the smallest value of $a / \lambda_{1}$ ) for which the asymptotic solution can be used. If the inequality is not satisfied, the solution may still be obtained. However, the terms of the resulting series oscillate and eventually increase so that it is not possible to determine where to truncate the series for maximum accuracy.

The asymptotic series (15) yields a countable infinite number of roots, each corresponding to a value $\eta_{0}$. In summing the residue series only the first few, and in many cases only the first root will be important since each represents a wave decaying exponentially at a rate determined by the imaginary part of the root.

Figure 2 is a plot according to (15) of the locations of the first root corresponding to $\eta_{0}=2.33810741$. The diagonal curves connecting the calculated points show the root loci as the ratio $a / \lambda_{1}$ is varied for fixed values of $N$ from 0 to 0.9 . The terminal point of each curve of constant $N$ is fixed by the indicated minimum allowed value of $y$. The vertical curves connecting the same points are the loci as $N$ is varied for fixed values of $y\left(\mathrm{Na} / \lambda_{2}\right.$ is constant). One of these loci $(y=16)$ is plotted to a linear scale as the lower left-hand member of Figure 3.

In region 5 of Figure 1, the same procedure yields the roots:

$$
\begin{aligned}
\nu=x & -\eta_{0} e^{\pi i / 3}\left(\frac{x}{2}\right)^{1 / 3}+\frac{\eta_{0}^{2}}{60} e^{2 \pi i / 3}\left(\frac{2}{x}\right)^{1 / 3}-\left[\frac{\eta_{0}^{3}}{1400}+\frac{1}{140}\right]\left(\frac{2}{x}\right) \\
& +e^{\pi / 3}\left[\frac{281 \eta_{0}^{4}}{4536000}+\frac{29 \eta_{0}}{12600}\right]\left(\frac{2}{x}\right)^{5 / 3} \\
& +\left(1-N^{2}\right)^{-1 / 2} \\
& +\frac{\eta_{0}}{6} e^{\pi i / 3}\left\{\left(1-N^{2}\right)^{-3 / 2}-\left(1-N^{2}\right)^{-1 / 2}\right\}\left(\frac{2}{x}\right)^{2 / 3} \\
& +\frac{\eta_{0}^{2}}{20} e^{2 \pi i / 3}\left\{\frac{3}{2}\left(1-N^{2}\right)^{-5 / 2}-\frac{13}{9}\left(1-N^{2}\right)^{-3 / 2}-\frac{1}{18}\left(1-N^{2}\right)^{-1 / 2}\right\}\left(\frac{2}{x}\right)^{4 / 3} \\
& +\frac{\eta_{0}}{36} e^{\pi i / 3}\left\{\left(1-N^{2}\right)^{-3}-2\left(1-N^{2}\right)^{-2}+\left(1-N^{2}\right)^{-1}\right\}\left(\frac{2}{x}\right)^{5 / 3} \\
& -\frac{1}{8}\left\{\frac { \eta _ { 0 } ^ { 3 } } { 2 } \left[\frac{5}{7}\left(1-N^{2}\right)^{-7 / 2}-\frac{21}{25}\left(1-N^{2}\right)^{-5 / 2}+\frac{23}{175}\left(1-N^{2}\right)^{-3 / 2}\right.\right. \\
& \left.-\frac{1}{175}\left(1-N^{2}\right)^{-1 / 2}\right]-\left[\frac{5}{28}\left(1-N^{2}\right)^{-7 / 2}-\frac{3}{10}\left(1-N^{2}\right)^{-5 / 2}\right. \\
& \left.\left.+\frac{13}{140}\left(1-N^{2}\right)^{-3 / 2}+\frac{1}{35}\left(1-N^{2}\right)^{-1 / 2}\right]\right\}\left(\frac{2}{x}\right)^{2}+0\left(x^{-7 / 3}\right) .
\end{aligned}
$$

As $y$ tends to zero $(N \rightarrow 0)$ all terms containing $N$ except the first approach zero, and the roots approach the limit, $\nu=\nu_{x}+1$ where $\nu_{x}$ is the solution of $H_{\nu}^{(1)}(x)=0$. As with (15), the development of (16) requires the power series expansion of $\left[1-\eta_{0} e^{\pi i / 3} / \gamma_{0}^{\prime 2}\right]^{1 / 2}$, where $\gamma_{0}^{\prime}=\left(1-N^{2}\right)^{1 / 2}(x / 2)^{1 / 3}$, so the solution is correct only for $\gamma_{0}^{\prime 2}>\left|\eta_{0}\right|$.

Root loci corresponding to (16) are plotted in Figure 4 for the first value of $\eta_{0}$. In 


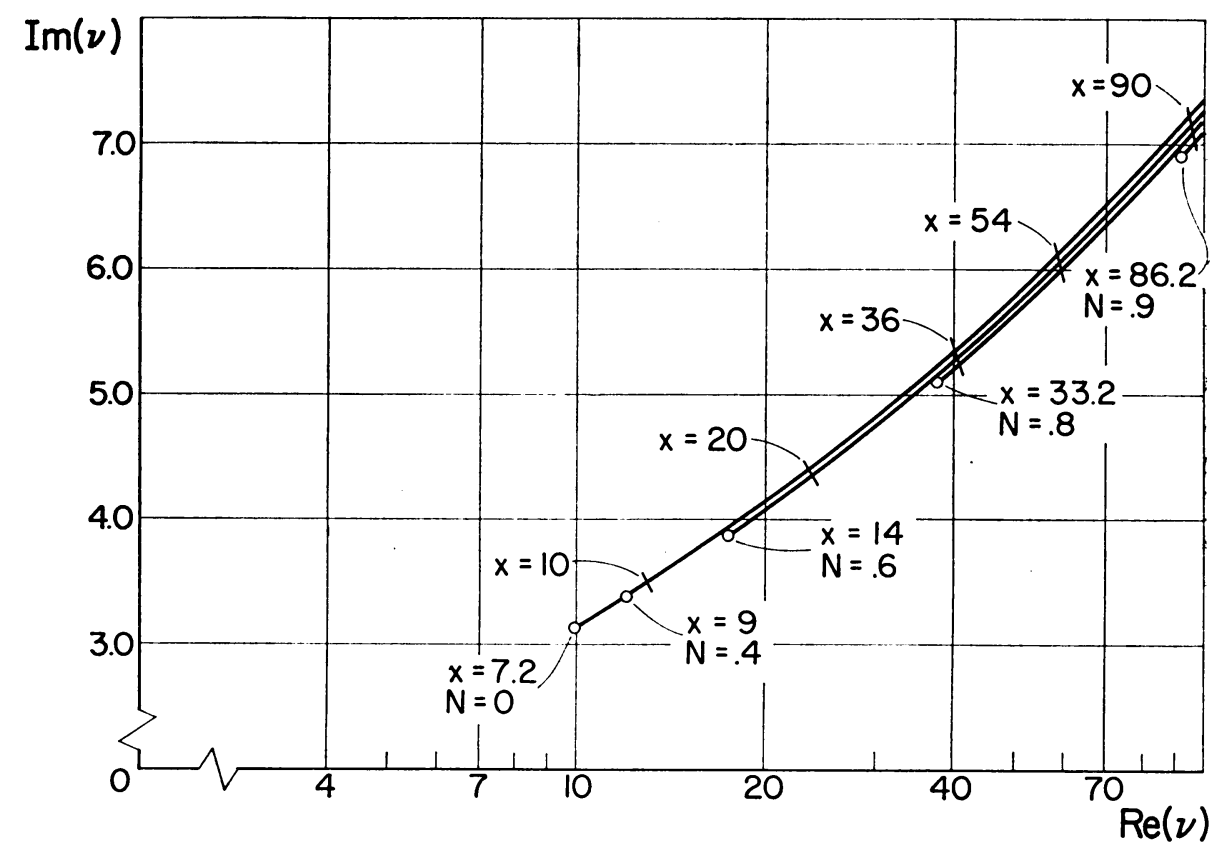

FIG. 4. The first root of $x \frac{H_{\nu}^{(1)^{\prime}}(x)}{H_{\nu}^{(1)}(x)}-y \frac{H_{\nu}^{(2)^{\prime}}(y)}{H_{\nu}^{(2)}(y)}=0$ near $|\nu|=x$.

its domain of applicability this formula is much less sensitive to the value of $N$ than the expansion for the roots near $y$. On the linear scale of Figure 5 the four points bunched on the upper segment of the curve correspond to the variation of $N$ from 0 to 0.6 , which is all that the selected value, $x=16$, will allow in the regime, $0(x-y)=0(x)$.

Case 2: $x-y=0\left(z^{1 / 3}\right)$. When regions 4 and 5 overlap, equation (1) may be expanded in a Taylor series about either $x$ or $y$. We begin by expanding $x H_{\nu}^{(1)}(x) / H_{\nu}^{(1)}(x)$ about $y$, which yields

$$
x \frac{H_{\nu}^{(1)^{\prime}}(x)}{H_{\nu}^{(1)}(x)}=y \frac{H_{\nu}^{(1)^{\prime}}(y)}{H_{\nu}^{(1)}(y)}+z \frac{\partial}{\partial y}\left\{y \frac{H_{\nu}^{(1)}{ }^{\prime}(y)}{H_{\nu}^{(1)}(y)}\right\}+\frac{z^{2}}{2 !} \frac{\partial^{2}}{\partial y^{2}}\left\{y \frac{H_{\nu}^{(1)^{\prime}}(y)}{H_{\nu}^{(1)}(y)}\right\}+\cdots
$$

with $z=x-y$. By means of the Wronskian relation and (17) equation (1) becomes

$$
-\frac{4 i}{H_{\nu}^{(1)}(y) H_{\nu}^{(2)}(y)}=z \frac{\partial}{\partial y}\left\{y \frac{H_{\nu}^{(1)^{\prime}}(y)}{H_{\nu}^{(1)}(y)}\right\} \frac{z^{2}}{2 !} \frac{\partial^{2}}{\partial y^{2}}\left\{y \frac{H_{\nu}^{(1)}(y)}{H_{\nu}^{(1)}(y)}\right\}+\cdots .
$$

For small values of $z$ solutions of (18) are possible only when $0(|\nu-x|)=$ $0(|\nu-y|)=0(x)$. The appropriate expansions are (A.4b) and (A.7). Substitution in (18) and rearrangement yields

$$
\begin{aligned}
\exp & \left\{2\left(\nu^{2}-y^{2}\right)^{1 / 2}-2 \nu \cosh ^{-1}(\nu / y)-i \pi / 2\right\} \\
& =\frac{z y}{2\left(\nu^{2}-y^{2}\right)}\left\{1+\frac{17 \nu^{2}}{12\left(\nu^{2}-y^{2}\right)^{3 / 2}}-\frac{1}{4\left(\nu^{2}-y^{2}\right)^{1 / 2}}+\frac{z \nu^{2}}{2 y\left(\nu^{2}-y^{2}\right)}+0\left(y^{-2}\right)\right\} .
\end{aligned}
$$

We shall seek an asymptotic solution of the form

$$
\nu=\alpha y+i \omega,
$$




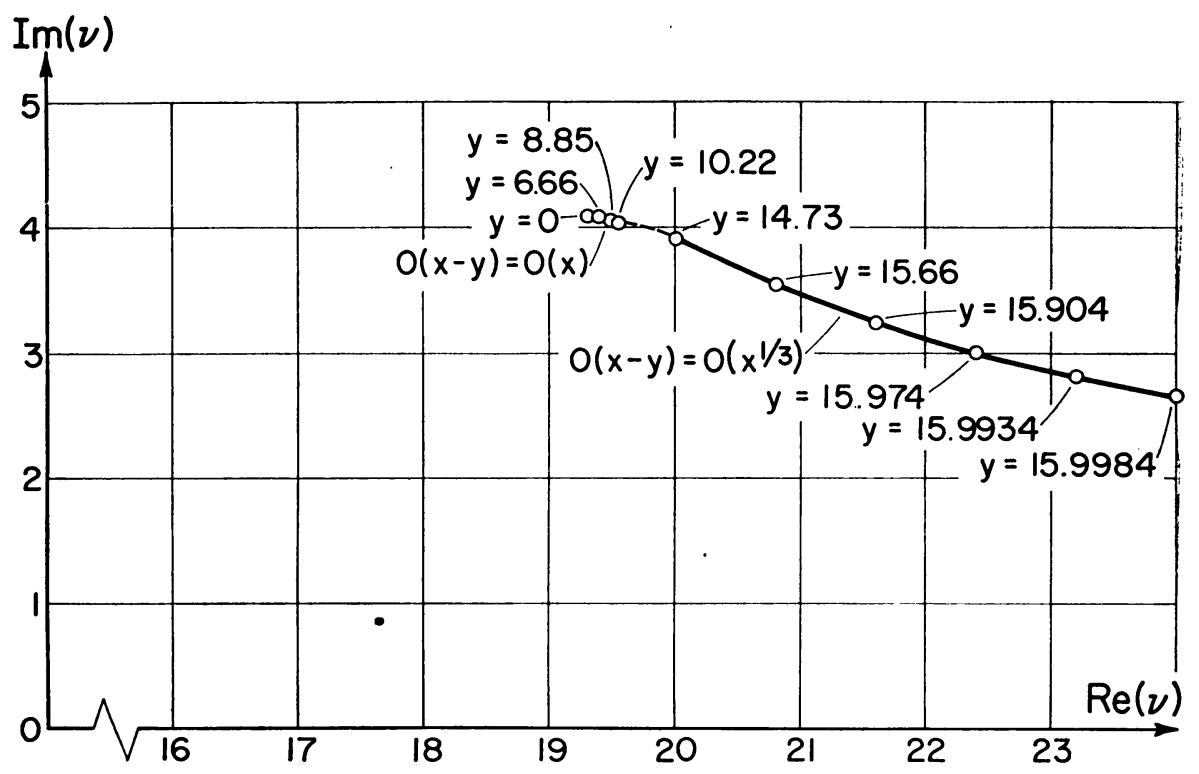

Fig. 5. Locus of the first root near $|\nu|=x$,

$$
x \frac{H_{\nu}^{(1)}(x)}{H_{v}^{(1)}(x)}-y \frac{H_{v}^{(2) '}(y)}{H_{v}^{(2)}(y)}=0 \text { for } x=16 .
$$

where $\alpha=$ const. and

$$
\omega=b_{0}+b_{1} / y+0\left(y^{-2}\right)=0(1)
$$

$b_{0}$ and $b_{1}$ are functions of $\alpha$ and $z$ only. Substituting (20a) in (19) and changing variables to $\Omega=\left(\alpha^{2}-1\right)^{1 / 2} y$ and $T=\alpha+\left(\alpha^{2}-1\right)^{1 / 2}$ gives

$$
\begin{aligned}
\exp \left\{2 \Omega+\omega^{2} y^{2} / \Omega^{3}-2 \alpha y \ln T-\alpha \omega^{2} y^{2} / \Omega^{2} T-\alpha y \omega^{2} / \Omega^{2}\right. \\
\left.+2 \omega^{2} / \Omega+2 i \alpha \omega y / \Omega-2 i \omega \ln T-2 i \omega \alpha y / \Omega-i \pi / 2+0\left(y^{-2}\right)\right\} \\
=\left(z y / 2 \Omega^{2}\right)\left\{1+17 \alpha^{2} y^{2} / 12 \Omega^{3}-1 / 4 \Omega+z \alpha^{2} y / 2 \Omega^{2}+0\left(y^{-2}\right)\right\} \\
\quad \cdot \exp \left\{-2 i \alpha \omega y / \Omega^{2}+0\left(y^{-2}\right)\right\} .
\end{aligned}
$$

Equating phases, we find with help of (20b) that

$-2 b_{0} \ln T-2 \frac{b_{1}}{y} \ln T-\pi / 2=-2 \alpha b_{0} /\left(\alpha^{2}-1\right)^{1 / 2} y+2 \pi n+0\left(y^{-2}\right)$

$$
n=0, \pm 1, \pm 2, \cdots \text {. }
$$

The equality of the magnitudes leads to

$$
\begin{gathered}
\exp \left\{2 y\left[\left(\alpha^{2}-1\right)^{1 / 2}-\alpha \ln T\right]+b_{0}^{2} /\left(\alpha^{2}-1\right)^{1 / 2} y+2 b_{0} b_{1} /\left(\alpha^{2}-1\right)^{1 / 2} y^{2}+0\left(y^{-3}\right)\right\} \\
=\frac{z}{2\left(\alpha^{2}-1\right) y}\left\{1-\frac{1}{4\left(\alpha^{2}-1\right)^{1 / 2} y}+\frac{17 \alpha^{2}}{12\left(\alpha^{2}-1\right)^{3 / 2} y}+\frac{z \alpha^{2}}{2\left(\alpha^{2}-1\right) y}+0\left(y^{-2}\right)\right\} .
\end{gathered}
$$


It follows from (22a) that

$$
b_{0}=-\frac{\pi / 4+n \pi}{\ln T}, \quad n=0,1,2 \cdots
$$

and

$$
b_{1}=\frac{\alpha b_{0}}{\left(\alpha^{2}-1\right)^{1 / 2} \ln T} .
$$

It remains only to determine corresponding values of $\alpha$ and $z$ through (22b). The roots in the lower half-plane are then given by equation (20a).

In the upper half-plane equation (1) is approximated by expanding about the point $y=x$. Thus,

$$
-\frac{4 i}{\pi H_{\nu}^{(1)}(x) H_{\nu}^{(2)}(x)}=z \frac{\partial}{\partial x}\left\{x \frac{H_{\nu}^{(2)^{\prime}}(x)}{H_{\nu}^{(2)}(x)}\right\}-\frac{z^{2}}{2 !} \frac{\partial^{2}}{\partial z^{2}}\left\{x \frac{H_{\nu}^{(2)}{ }^{\prime}(x)}{H_{\nu}^{(2)}(x)}\right\}+\cdots .
$$

However, when $\nu$ is in region 3 (Figure 1),

$$
\frac{H_{\nu}^{(2)^{\prime}}(x)}{H_{\nu}^{(2)}(x)} \sim \frac{H_{\nu}^{(1)}(x)}{H_{\nu}^{(1)}(x)}
$$

so that (24) and (18) are identical if, in the latter, $y$ is replaced by $x$ and the signs of $z^{2}, z^{4}, z^{6}$, etc. are changed. Thus $b_{0}$ and $b_{1}$, which do not depend on $z$, are unchanged, except that $n=-1,-2,-3 \cdots$ in (23a) and the roots above the axis are

$$
\nu=\alpha x+i\left[b_{0}+b_{1} / x+0\left(x^{-2}\right)\right] .
$$

The only other modification is in (22b) where $x$ replaces $y$ and the sign of the quadratic $z$ term is changed.

The solutions are accurate only when the expansions of equation (1) converge with sufficient rapidity. Their rapid convergence requires $z<1$, and either $\nu^{2}\left(\nu^{2}-y^{2}\right)^{-3 / 2} \ll 1$ or $\nu^{2}\left(\nu^{2}-x^{2}\right)^{-3 / 2} \ll 1$.

The right-hand segments of Figures 3 and 5 are the loci of the initial root calculated in this way for $y=16$ and $x=16$ respectively, while Figure 2 presents curves for $N=.99$, .999 , and .9999 .

3. Concluding remarks. The solutions obtained here apply for real $x$ and $y$. If $y$ has a small positive imaginary part (lossy dielectric) the results of case 1 are unchanged. In case $2, z=x-y$ is a non-zero complex number and the roots do not migrate to infinity. For $|z| \ll 1$, however, the roots would have very large real parts. Their loci would not be easy to find because of the complex character of $z$.

Franz and Beckmann [9], discuss qualitatively the solution of equation (1) in the first quadrant with $y$ complex and $|y|>x$. They conclude that the roots behave in a way similar to that found here for case 1 . They state further that as $x \rightarrow y$ the roots approach the solutions of $H_{\nu}^{(1)}(x)=0$; this conclusion, as we have seen, is incorrect. The error they have made is to assume that $\left(1-\nu^{2} / y^{2}\right)^{1 / 2}$ may always be approximated by $\left(1-x^{2} / y^{2}\right)^{1 / 2}=\left(1-1 / N^{2}\right)^{1 / 2}$. This is surely not the case when $x \rightarrow y$ or $N \rightarrow 1$.

\section{Appendix A: Properties of Hankel Functions}

The Hankel functions, $H_{\nu}^{(1)}(x)$ and $H_{\nu}^{(2)}(x)$, are two linearly independent solutions of Bessel's differential equation which satisfy the relations 


$$
\begin{gathered}
H_{-\nu}^{(1)}(x)=e^{i \nu \pi} H_{\nu}^{(1)}(x) \\
H_{-\nu}^{(2)}(x)=e^{-i \nu \pi} H_{\nu}^{(2)}(x) .
\end{gathered}
$$

The Debye asymptotic formulae, [14]-[15], for the Hankel functions are valid for $0(|\nu-x|)=0(x)$. For real $x$ various expressions represent the Hankel functions in different regions of the $\nu$ plane. The regions are shown in Figure 6 and the curves separating them are defined by

$$
\operatorname{Im}(x \sin \varphi-\nu \varphi)=0
$$

or

$$
\operatorname{Im}\left[\left(x^{2}-\nu^{2}\right)^{1 / 2}-\nu \cos ^{-1} \nu / x\right]=0
$$

where

$$
\nu=x \cos \varphi .
$$

The roots of $H_{v}^{(1)}(x)=0$ and $H_{v}^{(1)^{\prime}}(x)=0$ lie on the solid curves while those of $H_{v}^{(2)}(x)=0$ and its derivative are situated on the broken curves [16].

The asymptotic forms:

Regions I and II

$$
\begin{aligned}
& H_{\nu}^{(1)}(x) \sim\left(\frac{2}{\pi}\right)^{1 / 2} \frac{\exp \left\{i\left(x^{2}-\nu^{2}\right)^{1 / 2}-i \nu \cos ^{-1} \nu / x-i \pi / 4\right\}}{\left(x^{2}-\nu^{2}\right)^{1 / 4}} \\
& \cdot\left\{1-\frac{i}{\left(x^{2}-\nu^{2}\right)^{1 / 2}}\left(\frac{1}{8}-\frac{5 \nu^{2}}{24\left(\nu^{2}-x^{2}\right)}\right)+0\left(x^{-2}\right)\right\} \\
& H_{\nu}^{(2)}(x) \sim\left(\frac{2}{\pi}\right)^{1 / 2} \frac{\exp \left\{-i\left(x^{2}-\nu^{2}\right)^{1 / 2}+i \nu \cos ^{-1} \nu / x+i \pi / 4\right\}}{\left(x^{2}-\nu^{2}\right)^{1 / 4}} \\
& \cdot\left\{1+\frac{i}{\left(x^{2}-\nu^{2}\right)^{1 / 2}}\left(\frac{1}{8}-\frac{5 \nu^{2}}{24\left(\nu^{2}-x^{2}\right)}\right)+0\left(x^{-2}\right)\right\}
\end{aligned}
$$

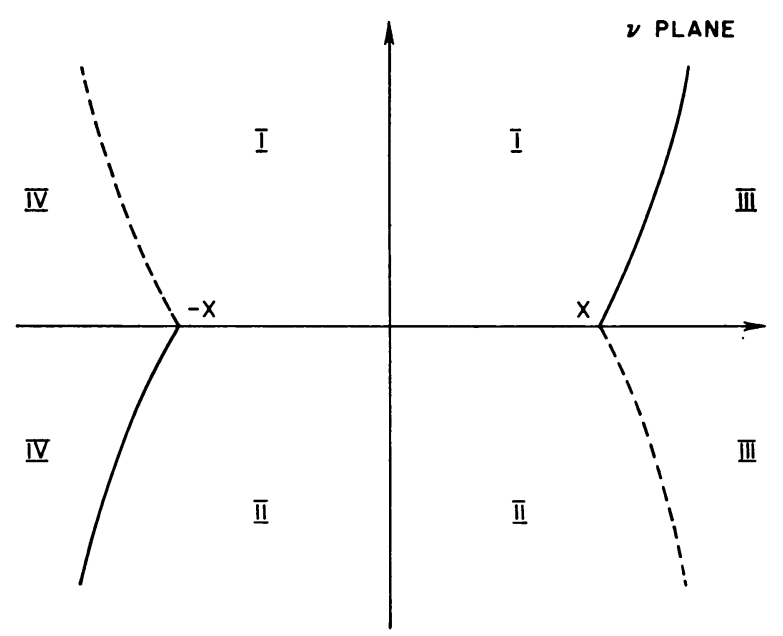

FIG. 6. Regions in the $\nu$ plane for Hankel functions. 
with

$$
\begin{aligned}
& \left|\arg \left(x^{2}-\nu^{2}\right)^{1 / 2}\right|<\pi / 2 \\
& 0 \leq \operatorname{Re}\left(\cos ^{-1} \nu / x\right) \leq \pi \\
& \operatorname{Im}\left(\cos ^{-1} \nu / x\right)=\left\{\begin{array}{l}
<0 \text { I } \\
=0 \text { boundary } \\
>0 \text { II }
\end{array}\right.
\end{aligned}
$$

Region III

$$
\begin{gathered}
H_{\nu}^{(1)}(x) \sim-H_{\nu}^{(2)}(x) \\
H_{\nu}^{(1)}(x) \sim-i\left(\frac{2}{\pi}\right)^{1 / 2} \frac{\exp \left\{-\left(\nu^{2}-x^{2}\right)^{1 / 2}+\nu \cosh ^{-1} \nu / x\right\}}{\left(\nu^{2}-x^{2}\right)^{1 / 4}} \\
\cdot\left\{1-\frac{1}{\left(\nu^{2}-x^{2}\right)^{1 / 2}}\left(\frac{1}{8}-\frac{5 \nu^{2}}{24\left(\nu^{2}-x^{2}\right)}\right)+0\left(x^{-2}\right)\right\}
\end{gathered}
$$

with

$$
\begin{aligned}
\left|\arg \left(\nu^{2}-x^{2}\right)^{1 / 2}\right| & <\pi / 2 \\
\operatorname{Re}\left(\cosh ^{-1} \nu / x\right) & >0 \\
\left|\operatorname{Im}\left(\cosh ^{-1} \nu / x\right)\right| & <\pi / 2 .
\end{aligned}
$$

Region IV

Application of (A.1a) and (A.1b) to the results for region III yield the desired forms.

If $H_{v}^{(1)}(x)$ is required in the vicinity of the solid curves, the formulae appropriate to the two adjacent regions must be added. Thus near the curve separating I and III the result is

$$
H_{\nu}^{(1)}(x) \sim 2 i\left(\frac{2}{\pi}\right)^{1 / 2} \frac{\sin \left\{\left(x^{2}-\nu^{2}\right)^{1 / 2}-\nu \cos ^{-1} \nu / x-\pi / 4\right\}}{\left(x^{2}-\nu^{2}\right)^{1 / 4}}\left[1+0\left(x^{-1}\right)\right],
$$

which it is interesting to compare with (A.2b). As $\nu$ moves away from the boundary one of the two exponential functions dominates to give the forms previously mentioned. In the vicinity of the broken curves both forms of $H_{v}^{(1)}(x)$ are identical, but $H_{v}^{(2)}(x)$ is expressed as in (A.5). At the solid curves the formulae representing $H_{\nu}^{(2)}(x)$ are continuous.

The ratios appearing in equation (1) can now be approximated in that part of the $\nu$-plane for which $0(|\nu-x|)=0(x)$ as follows:

Regions I and II

$x \frac{H_{\nu}^{(1)}(x)}{H_{\nu}^{(1)}(x)} \sim i\left(x^{2}-\nu^{2}\right)^{1 / 2}-\frac{x^{2}}{2\left(x^{2}-\nu^{2}\right)}+\frac{i x^{2}}{8\left(x^{2}-\nu^{2}\right)^{3 / 2}}\left(1-\frac{5 \nu^{2}}{\nu^{2}-x^{2}}\right)+0\left(x^{-2}\right)$

Region III

$$
\begin{aligned}
x \frac{H_{\nu}^{(1)}(x)}{H_{\nu}^{(1)}(x)} \sim x H_{\nu}^{H_{\nu}^{(2)}(x)} & \sim-\left(\nu^{2}-x^{2}\right)^{1 / 2} \\
& +\frac{x^{2}}{2\left(\nu^{2}-x^{2}\right)}-\frac{x^{2}}{8\left(\nu^{2}-x^{2}\right)^{3 / 2}}\left(1-\frac{5 \nu^{2}}{\left(\nu^{2}-x^{2}\right)}\right)+0\left(x^{-2}\right) .
\end{aligned}
$$


When $0(|\nu-x|)=0\left(x^{1 / 3}\right)$, the Schöbe [17] asymptotic forms must be used

$$
H_{\nu}^{(1),(2)}(x) \sim 2\left(\frac{2}{x}\right)^{1 / 3} p(x, \nu) e^{* \pi, i / 3} A i\left[-q(x, \nu) e^{* \pi i / 3}\right]+0\left(x^{-2 / 3(m+1)}\right),
$$

where the upper sign goes with $H_{\nu}^{(1)}(x)$. For both Hankel functions

$$
p(x, \nu)=\sum_{j=0}^{m}(-1)^{i} P_{i}(\xi)\left(\frac{2}{x}\right)^{2 i / 3}
$$

and

$$
q(x, \nu)=\sum_{k=0}^{m}(-1)^{k} Q_{k}(\xi)\left(\frac{2}{x}\right)^{2 k / 3}
$$

where $\xi=(\nu-x)(2 / x)^{1 / 3}$ and

$$
\begin{aligned}
P_{0}(\xi) & =1 & Q_{0}(\xi) & =\xi \\
P_{1}(\xi) & =\frac{1}{15} \xi & Q_{1}(\xi) & =\frac{1}{60} \xi^{2} \\
P_{2}(\xi) & =\frac{13}{1260} \xi^{2} & Q_{2}(\xi) & =\frac{2}{1575} \xi^{3}+\frac{1}{140} \\
P_{3}(\xi) & =\frac{109}{56700} \xi^{3}+\frac{1}{900} & Q_{3}(\xi) & =\frac{41}{283500} \xi^{4}+\frac{4}{1575} \xi .
\end{aligned}
$$

For $0(|\nu-x|)=0\left(x^{1 / 3}\right)$,

$$
x \frac{H_{\nu}^{(1),(2)}(x)}{H_{\nu}^{(1),(2)}(x)} \sim-\frac{1}{3}+x \frac{\partial_{x} p}{p}-x e^{* \pi i / 3} \partial_{x} q \frac{A i^{\prime}\left(-q e^{\mp i / 3}\right)}{A i\left(-q e^{\mp \pi i / 3}\right)}+0\left(x^{-2 m / 3}\right),
$$

where $\partial_{x} q$ and $\partial_{x} p$ are derivatives with respect to $x$ and $A i^{\prime}(\eta)=d A i(\eta) / d \eta$.

Appendix B: The Airy Functions

The Airy functions are solutions of

$$
f^{\prime \prime}(\eta)-\eta f(\eta)=0 .
$$

$A i(\eta)$ is the only solution which approaches zero as $\eta \rightarrow \pm \infty$, [12], while $B i(\eta)$ is a linearly independent solution. $A i(\eta)$ satisfies the relations

$$
\begin{aligned}
& A i(w \eta)=-\frac{1}{2} w^{2}[A i(\eta)-i B i(\eta)] \\
& A i\left(w^{2} \eta\right)=-\frac{1}{2} w[A i(\eta)+i B i(\eta)]
\end{aligned}
$$

where

$$
w=e^{2 \pi i / 3} .
$$

Through (B.1) the derivatives of $A i(\eta)$ and $B i(\eta)$ are expressed as polynomials in $\eta$ multiplied by the function and its first derivative. The first eight derivatives are given:

$$
\begin{aligned}
& A i^{(2)}(\eta)=\eta A i(\eta) \\
& A i^{(3)}(\eta)=A i(\eta)+\eta A i^{\prime}(\eta)
\end{aligned}
$$




$$
\begin{aligned}
& A i^{(4)}(\eta)=\eta^{2} A i(\eta)+2 A i^{\prime}(\eta) \\
& A i^{(5)}(\eta)=4 \eta A i(\eta)+\eta^{2} A i^{\prime}(\eta) \\
& A i^{(6)}(\eta)=\left(4+\eta^{3}\right) A i(\eta)+6 \eta A i^{\prime}(\eta) \\
& A i^{(7)}(\eta)=9 \eta^{2} A i(\eta)+\left(10+\eta^{3}\right) A i^{\prime}(\eta) \\
& A i^{(8)}(\eta)=\left(28 \eta+\eta^{4}\right) A i(\eta)+12 \eta^{2} A i^{\prime}(\eta) .
\end{aligned}
$$

The zeros of $A i(\eta), B i(\eta)$, and their first derivatives lie on the negative real axis. The first five solutions of $A i\left(\eta_{0}\right)=0$ are

$$
\begin{aligned}
\eta_{0}= & -2.33810741 \\
& -4.08794944 \\
& -5.52055983 \\
& -6.78670809 \\
& -7.94413359 .
\end{aligned}
$$

\section{REFERENCES}

[1] T. T. Wu, Phys. Rev. 104, 1201 (1956).

[2] G. N. Watson, Proc. Roy. Soc. 95, 83 (1918).

[3] E. C. Titchmarsh, Eigenfunction expansions, (Oxford, 1958).

[4] N. Marcuvitz, Commun. Pure Appl. Math. 4, 284 (1951).

[5] W. Franz and K. Deppermann, Annalen Phys. 10, 361 (1952).

[6] W. Franz, Z. Naturf. 9a, 705 (1954).

[7] W. Franz and R. Galle, Z. Naturf. 10a, 374 (1955).

[8] P. Beckmann and W. Franz, Z. Naturf. 12a, 257 (1957).

[9] P. Beckmann and W. Franz, IRE AP-4, 3 (1956).

[10] S. I. Rubinow, NYU Res. Rpt. EM-163 (December 1960).

[11] A. Erdelyi, Asymptotic expansions, (Dover, 1956).

[12] J. C. P. Miller, Airy integral, (Brit. Assoc. Adv. of Sci., Math Tables, Part-vol. B, 1946).

[13] A. Sommerfeld, Partial differential equations, (Academic, 1949).

[14] P. Debye, Math. Ann. 67, 535 (1909).

[15] G. N. Watson, Treatise on the theory of bessel functions, (Cambridge, 1958).

[16] P. Beckmann and W. Franz, Z. Angew. Math. Mech. 37, 17 (1957).

[17] W. Schöbe, Acta Math. 92, 265 (1954). 